GANIT J. Bangladesh Math. Soc. (ISSN 1606-3694) 37 (2017) 147-159

\title{
GENERALIZED GALERKIN FINITE ELEMENT FORMULATION FOR THE NUMERICAL SOLUTIONS OF SECOND ORDER NONLINEAR BOUNDARY VALUE PROBLEMS
}

\author{
Hazrat Ali* and Md. Shafiqul Islam \\ Department of Applied Mathematics, University of Dhaka, Dhaka-1000, Bangladesh \\ *Corresponding author: naim2010math@gmail.com
}

Received 13.07.2017 Accepted 26.10.2017

\begin{abstract}
We use Galerkin finite element method (GFEM) to solve second order linear and nonlinear boundary value problems (BVPs). First we develop FEM formulation for a class of linear and nonlinear BVPs. Then we present convergence analysis of the method. Later, we give the solution of some nonlinear BVPs with Diritchlet, Neumann and Robin boundary conditions. All results are compared with the exact solution and sometimes with the results of the existing method to verify the convergence, stability and consistency of this method. The results are depicted graphically as well as in the tabular form.
\end{abstract}

Keyword: Galerkin finite element method, nonlinear boundary value problem.

\section{Introduction}

Numerical methods play a vital role in science and engineering in terms of solving and analyzing problems. Solutions to scientific and engineering problems can be achieved more easily by numerical method with the help of computers. So the importance of numerical analysis is increasing day by day, because most of the natural phenomena can be described by differential equations with varying boundary conditions easily, but the solutions of which cannot be obtained analytically except very simple cases. To solve these problems, we use several methods such as Finite Difference Method (FDM), Galerkin Method, Collocation Method, Least Square Method, Sub-domain Method [1], Adomian Decomposition Method [2], Shooting Method [3] etc.

Prior to its conception, the finite difference method held a dominant position in the numerical solution of continuum problem. But it gives value at particular points only and cannot be used to evaluate the values at the desired points between two grid points. Without that it takes more computational cost for getting higher accuracy. For this limitations of the FDM, people start to use Galerkin Method, Collocation Method and Least Square Method for solving differential equations. These methods employ trial functions which must satisfy the boundary conditions. This is little easy in simple problems but in real life problems it is too tough to find these types of trial functions. 
So in recent years, Galerkin Finite Element Method (GFEM) [5] is becoming very much popular technique for obtaining approximate solutions to the ordinary differential equations and the partial differential equations that arise in science and engineering applications. Because GFEM gives a polynomial at each point instead of value, so it can give value at any point within the domain. To apply GFEM, no need to convert the boundary value problems into initial value problems. In this method one can easily use the finite element shape functions instead of trial functions. It is a general technique for constructing approximate solutions to the boundary value problems. For this reason GFEM is widely used in solving differential equations.

Islam et al. [4] use Galerkin finite element method for solving initial boundary value problems of differential equations. Bhatti and Bracken [6] solved the nonlinear BVP with only Diritchlet boundary conditions which is limited within first order, where Islam and Shirin [7] solved the linear and nonlinear boundary value problems by using Galerkin Method with the help of Bernoulli polynomials.

To the best of our knowledge, none have solved the nonlinear boundary value problems with all boundary conditions by Galerkin finite element method yet. So in this paper, our main concern is to solve the nonlinear boundary value problems with all boundary conditions by using Galerkin finite element method.

\section{Finite Element Formulation for Second Order Linear BVPs}

Let us consider the general second order boundary value problem [5]

$$
-\frac{d}{d x}\left(p(x) \frac{d u}{d x}\right)+q(x) u(x)=f(x), \quad a \leq x \leq b
$$

whose boundary conditions are

$$
\begin{array}{ll}
u(a)=r_{1} & \left(p(x) \frac{d u}{d x}\right)_{\{x=a\}}=\tau_{1} \\
u(b)=r_{2} & \left(p(x) \frac{d u}{d x}\right)_{\{x=b\}}=\tau_{2}
\end{array}
$$

where $u(a)$ or $\tau_{1}$ and $u(b)$ or $\tau_{2}$ are specified numbers. Let us divide the domain $a \leq x \leq$ $b$ into $n$ sub-domains. Fig 1 shows the domain that is partitioned into $n$ elements of equal (not necessary) length. The nodes $i=1,2,3, \ldots \ldots, N$ and the corresponding $x_{i}$ are numbered sequentially from left to right starting with element [1]. The total number of degrees of freedom $N=(\gamma-1) \times n+1$, where $\gamma$ is the number of nodes in each element and $n$ is the number of elements in whole domain. The elements are numbered in a similar manner additionally with parenthesis $[e]$. 


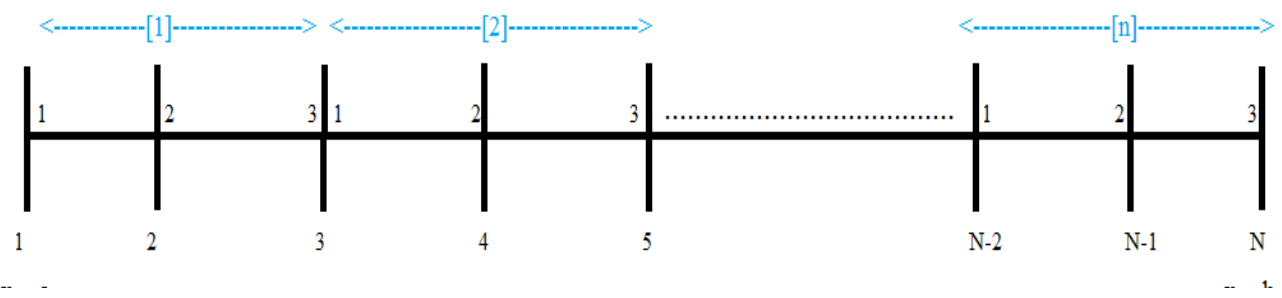

Fig 1: Domain Discrimination for Quadratic Shape Function.

Let the typical trial solution of an element $[e]$ be given by

$\tilde{u}(x)=\sum_{j=1}^{n} a_{j} \phi_{j}(x)$

Here $x$ represents the independent variable in the problem. The functions $\phi_{j}(x)$ are known as the trial functions or standard basis functions [5]. The coefficients $a_{j}$ are to be determined, parameters (called degrees of freedom or generalized co-ordinate) since $\tilde{u}(x)$ is a function of $x$ as well as $a_{j}$.

Now the weighted residual equation for typical element $[e]$ of the pattern (1) is

$\int_{[e]}\left[-\frac{d}{d x}\left(p(x) \frac{d \tilde{u}}{d x}\right)+q(x) \tilde{u}(x)-f(x)\right] \phi_{i}(x) d x=0 \quad a \leq x \leq b$

Integrating first term by parts and after simple modification, we get the following form

$\sum_{j=1}^{n} a_{j} \int_{[e]}\left[p(x) \frac{d \phi_{i}}{d x} \frac{d \phi_{j}}{d x}+q(x) \phi_{i} \phi_{j}\right] d x=\int_{[e]} f(x) \phi_{i}(x) d x-\left[-p(x) \frac{d \tilde{u}}{d x} \phi_{i}\right]_{[e]}$

This is an element equation for the typical element $[e]$ may be written in the conventional matrix form as

$\left[K^{[e]}\right]\{a\}=\left\{F^{[e]}\right\}$

Where $K^{[e]}$ and $F^{[e]}$ are called the Stiffness matrix and the Load vector. The entries of $K^{[e]}$ and $F^{[e]}$ are

$K_{i, j}^{[e]}=\int_{[e]}\left[p(x) \frac{d \phi_{i}}{d x} \frac{d \phi_{j}}{d x}+q(x) \phi_{i} \phi_{j}\right] d x$

$F_{i}^{[e]}=\int_{[e]} f(x) \phi_{i}(x) d x-\left[-p(x) \frac{d \tilde{u}}{d x} \phi_{i}\right]_{[e]}$

If we take the quadratic shape functions, then the bilinear form and the linear functional are computed element by element as follows

$$
K^{[1]}=\left[\begin{array}{lll}
K_{1,1}^{1} & K_{1,2}^{1} & K_{1,3}^{1} \\
K_{2,1}^{1} & K_{2,2}^{1} & K_{2,3}^{1} \\
K_{3,1}^{1} & K_{3,2}^{1} & K_{3,3}^{1}
\end{array}\right], \quad K^{[2]}=\left[\begin{array}{lll}
K_{1,1}^{2} & K_{1,2}^{2} & K_{1,3}^{2} \\
K_{2,1}^{2} & K_{2,2}^{2} & K_{2,3}^{2} \\
K_{3,1}^{2} & K_{3,2}^{2} & K_{3,3}^{2}
\end{array}\right], \ldots \ldots \ldots, K^{[n]}=\left[\begin{array}{lll}
K_{1,1}^{n} & K_{1,2}^{n} & K_{1,3}^{n} \\
K_{2,1}^{n} & K_{2,2}^{n} & K_{2,3}^{n} \\
K_{3,1}^{n} & K_{3,2}^{n} & K_{3,3}^{n}
\end{array}\right]
$$




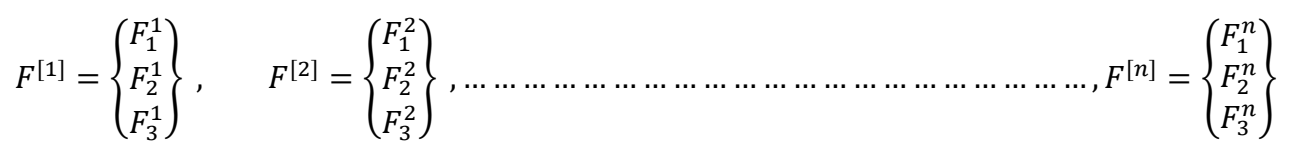

After computing for all elements and assembling, we finally get the system of equation of the form

$$
\left[\begin{array}{cccccccccc}
K_{1,1}^{1} & K_{1,2}^{1} & K_{1,3}^{1} & 0 & 0 & 0 & 0 & 0 & 0 & 0 \\
K_{2,1}^{1} & K_{2,2}^{1} & K_{2,3}^{1} & 0 & 0 & \vdots & \vdots & \vdots & \vdots & \vdots \\
K_{3,1}^{1} & K_{3,2}^{1} & K_{3,3}^{1}+K_{1,1}^{2} & K_{1,2}^{2} & K_{1,3}^{2} & \vdots & \vdots & \vdots & \vdots & \vdots \\
0 & 0 & K_{2,1}^{2} & K_{2,2}^{2} & K_{2,3}^{2} & \vdots & \vdots & \vdots & \vdots & \vdots \\
\vdots & \vdots & K_{3,1}^{2} & K_{3,2}^{2} & K_{3,3}^{2}+K_{1,1}^{3} & 0 & 0 & \vdots & \vdots & \vdots \\
\vdots & \vdots & 0 & 0 & 0 & \ddots & \ddots & \vdots & \vdots & \vdots \\
\vdots & \vdots & \vdots & \vdots & \vdots & \ddots & \ddots & 0 & 0 & 0 \\
\vdots & \vdots & \vdots & \vdots & \vdots & \vdots & 0 & K_{3,3}^{n-1}+K_{1,1}^{n} & K_{1,2}^{n} & K_{1,3}^{n} \\
\vdots & \vdots & \vdots & \vdots & \vdots & \vdots & \vdots & K_{2,1}^{n} & K_{2,2}^{n} & K_{2,3}^{n} \\
0 & \cdots & \cdots & \cdots & \cdots & 0 & 0 & K_{3,1}^{n} & K_{3,2}^{n} & K_{3,3}^{n}
\end{array}\right]\left[\begin{array}{c}
a_{1} \\
a_{2} \\
a_{3} \\
\vdots \\
\vdots \\
\vdots \\
\vdots \\
a_{N-1} \\
a_{N}
\end{array}\right]=\left[\begin{array}{c}
F_{1}^{1} \\
F_{2}^{1} \\
F_{3}^{1}+F_{1}^{2} \\
F_{2}^{2} \\
F_{3}^{2}+F_{1}^{3} \\
\vdots \\
\vdots \\
F_{3}^{n-1}+F_{1}^{n} \\
F_{2}^{n} \\
F_{3}^{n}
\end{array}\right]
$$

The resulting formal expression is called the element equations. Solving the system of equations (6), we will find the values of $a_{1}, a_{2}, a_{3}, \ldots \ldots \ldots, a_{N}$. Then putting these values into equation (3), we will obtain the piece-wise polynomial for each element.

The solution of equation (6) will be unique, if $K$ is a non-singular matrix. In order to ensure that $K$ is non-singular, the basis functions must be linearly independent. By definition, a set of functions $\phi_{j}(x)(j=1,2, \ldots, n)$ is linearly independent if

$$
\sum_{j=1}^{n} a_{j} \phi_{j}(x)=0
$$

implies that $a_{j}=0$ for $j=1,2, \ldots, n$. It is easy to show that $\phi_{j}(x)$ are linearly independent since no shape function can be expressed as the scaler multiple of other shape function.

\section{Finite Element Formulation for Second Order Non-linear BVPs}

Consider the radiation fin. The fin is assumed to liberate heat to its surrounding only through radiation. By using the one-dimensional form of the energy equation, the following nonlinear BVP is obtained for the solution of the temperature distribution [13]

$$
\begin{aligned}
& \frac{d^{2} U}{d R^{2}}+\left[\frac{1}{R+\rho}-\frac{\tan \alpha}{(1-R) \tan \alpha+\theta}\right] \frac{d U}{d R}-\frac{\beta U^{4}}{(1-R) \tan \alpha+\theta}=0, \quad 1 \leq R \leq 3 \\
& U(0)=1 \text { and }\left(\frac{d U}{d R}\right)_{R=1}=\frac{43}{3}
\end{aligned}
$$

Then following the above procedure, we finally get the compact form 


$$
\begin{aligned}
\sum_{j=1}^{n} a_{j} \int_{[e]}\left[\frac{d \phi_{i}}{d R} \frac{d \phi_{j}}{d R}\right. & -\left[\frac{1}{R+\rho}-\frac{\tan \alpha}{(1-R) \tan \alpha+\theta}\right] \phi_{i} \frac{d \phi_{j}}{d R} \\
& \left.+\frac{\beta}{(1-R) \tan \alpha+\theta} \phi_{i} \phi_{j}\left(\sum_{k=1}^{n} a_{k} \phi_{k}\right)^{3}\right] d R=\left[\phi_{i} \frac{d \widetilde{U}}{d R}\right]_{[e]}
\end{aligned}
$$

$i, j, k=1,2, \ldots \ldots \ldots \ldots n$

The above equation can be written for each element in the matrix form as

$$
\left[K^{[e]}+L^{[e]}\right] A^{[e]}=F^{[e]}
$$

Where the entries of the matrices $K^{[e]}, L^{[e]}, A^{[e]}$ and $F^{[e]}$ are $K_{i, j}^{[e]}, L_{i, j}^{[e]}, a_{j}$ and $F_{i}^{[e]}$ respectively given by

$K_{i, j}^{[e]}=\int_{[e]}\left[\frac{d \phi_{i}}{d R} \frac{d \phi_{j}}{d R}-\left[\frac{1}{R+\rho}-\frac{\tan \alpha}{(1-R) \tan \alpha+\theta}\right] \phi_{i} \frac{d \phi_{j}}{d R}\right] d R$

$L_{i, j}^{[e]}=\int_{[e]}\left[\frac{\beta}{(1-R) \tan \alpha+\theta} \phi_{i} \phi_{j}\left(\sum_{k=1}^{n} a_{k} \phi_{k}\right)^{3}\right] d R$

$F_{i}^{[e]}=\left[\phi_{i} \frac{d \tilde{u}}{d R}\right]_{[e]}$

where $i, j, k=1,2, \ldots \ldots \ldots, n$

Assembling those matrices following the above procedure, we find a nonlinear system of equation whose matrix form is

$$
[K+L]\{a\}=\{F\}
$$

which gives $N \times N$ stiffness matrix. For finding initial values of $a_{j}$, we neglect the nonlinear term $L$ from equation (11), then the equation (11) becomes

$$
[K]\{a\}=\{F\}
$$

Then we can find the initial values of coefficients $a_{j}$ solving equation (12) by the method described in the previous section. After getting the values of $a_{j}$, we substitute into equation (11) and starts Picard iteration [8]. The iteration process will continue until we find the desired accurate values of $a_{j}$. Then substituting the values of $a_{j}$ into equation (3), we get a piece-wise polynomial with variables $x$ for each element. Now we can compare this results with the exact results.

By using collocation method with the Haar wavelets, Siraj-ul-Islam et al. [13] have found a numerical result, which is given in Table 1 . Here we use $\rho=0.5, \alpha=60, \theta=0.05$ and $\beta=0.1$. From table 1, we observe in the present method, we just use only 31 nodes with 20 iterations, where Siraj-ul-Islam [13] used 256 nodes. 
Table 1: Numerical results of exact and approximate solutions of equation (7) using 15 quadratic elements and 20 iterations.

\begin{tabular}{|c|c|c|c|}
\hline $\mathbf{R}$ & $\begin{array}{c}\text { GFEM Solutions for 31 } \\
\text { Nodes }\end{array}$ & $\begin{array}{c}\text { Haar Solutions for 256 } \\
\text { Nodes [13] }\end{array}$ & $\begin{array}{c}\text { Na's Solutions by } \\
\text { Shooting Method [14] }\end{array}$ \\
\hline 0 & 1 & 1 & 1 \\
\hline 0.066667 & 0.963456166479602 & & \\
\hline 0.13333 & 0.931912530848484 & & 0.9044 \\
\hline 0.200000 & 0.904401279068787 & 0.90440 & \\
\hline 0.266667 & 0.880229197969537 & & 0.8400 \\
\hline 0.333333 & 0.858889116152377 & & \\
\hline 0.400000 & 0.840005387663005 & 0.84000 & \\
\hline 0.466667 & 0.823299799316353 & & \\
\hline 0.533333 & 0.808570102628505 & & \\
\hline 0.600000 & 0.795676981962007 & 0.79567 & \\
\hline 0.666667 & 0.784537299941699 & & \\
\hline 0.733333 & 0.775122793856479 & & \\
\hline 0.800000 & 0.767464535844083 & & \\
\hline 0.866667 & 0.761664843415977 & & \\
\hline 0.933333 & 0.757920551199750 & & \\
\hline 1.000000 & 0.756565900245134 & 0.75656 & \\
\hline & & & \\
\hline
\end{tabular}

\section{Convergence Analysis}

Let us consider the general second order boundary value problem

$-\frac{d}{d x}\left(p(x) \frac{d u}{d x}\right)+q(x) u(x)=f(x), \quad a \leq x \leq b$

with boundary conditions

$u(a)=u(b)=0$

To obtain an approximate solution of equation (13a), we construct a finite dimensional subspace of $E(I)$ and select a trial solution

$\tilde{u}(x)=\sum_{j=1}^{n} a_{j} \phi_{j}(x)$

where $\phi_{j}(x)$ satisfies the boundary conditions, i.e

$\phi_{i}(a)=\phi_{j}(b)=0$

The family of functions that can be written in this way will be denoted by $S(I)$. The functions $\phi_{j}(x)$, called basis functions, will be defined such that $S(I) \subset E(I)$, where, $E(I)$ is the 
energy space. Here the number $n$ is the dimension of $S(I), N$ denotes the number of elements and $l_{k}$ denotes the length of $k$ th element.

Our main goal is to minimize the error of approximate result in the energy norm. In the following discussion, we will denote the exact solution by $u_{E X}$ and the approximate solution by $u_{F E}$. We have to find $u_{F E} \epsilon \tilde{S}(I)$ such that $B\left(u_{F E}, v\right)=F(v)$ for all $v \in S_{0}(I)$. Where $B$ is a bilinear form on $\widetilde{S}(I) \times S_{0}(I)$.

\section{Theorem 1}

The error of approximation $e:=u_{E X}-u_{F E}$ is orthogonal to all test functions in $S_{0}(I)$ in the following sense [11]

$$
B(e, v)=0 \quad \forall v \in S_{0}(I)
$$

This is a basic property of the error of approximation, known as the Galerkin orthogonality.

\section{Proof}

Since $S_{0}(I) \subset E_{0}(I)$ then,

$B\left(u_{E X}, v\right)=F(v) \quad \forall v \in S_{0}(I)$

$B\left(u_{F E}, v\right)=F(v) \quad \forall v \in S_{0}(I)$

Subtracting (17) from (16), we get

$B\left(u_{E X}-u_{F E}, v\right)=0$

$\forall v \in S_{0}(I)$

$B(e, v)=0$

$\forall v \in S_{0}(I)$

Which is the equation (15).

\section{Theorem 2}

The GFEM will select the coefficients of the basis functions in such a way that the energy norm of the error $\|e\|_{E}$ will be minimum [11]. i.e.

$$
\left\|u_{E X}-u_{F E}\right\|_{E} \quad=\min \left\|u_{E X}-u\right\|_{E} \quad \forall u \in \tilde{S}(I)
$$

\section{Proof}

The error of approximate solution is $e:=u_{E X}-u_{F E}$.

For an arbitrary $v \in S_{0}(I),\|v\|_{E} \neq 0$, we have from the definition of energy norm

$$
\begin{aligned}
\|e+v\|_{E}^{2} & =\frac{1}{2} B(e+v, e+v) \\
& =\frac{1}{2} B(e, e)+\frac{1}{2} B(e, v)+\frac{1}{2} B(v, e)+\frac{1}{2} B(v, v) \\
& =\frac{1}{2} B(e, e)+B(e, v)+\frac{1}{2} B(v, v)
\end{aligned}
$$


Now since $B(e, v)=0 \forall v \in S_{0}(I)$ from the preceding theorem and

$$
\begin{aligned}
& \|v\|_{E} \neq 0 \\
& \Rightarrow \|\left. v\right|_{E} ^{2}>0 \\
& \Rightarrow \frac{1}{2} B(v, v)>0
\end{aligned}
$$

Then from equation (19), we get

$$
\begin{aligned}
& \left.\|e+v\|\right|_{E} ^{2}=\frac{1}{2} B(e, e)+\frac{1}{2} B(v, v) \\
& \left.\Rightarrow\|e+v\|\right|_{E} ^{2}>\|\left. e\right|_{E} ^{2} \\
& \left.\Rightarrow|| e\right|_{E} ^{2}<\left.\|e+v\|\right|_{E} ^{2} \\
& \Rightarrow|| u_{E X}-\left.u_{F E}\right|_{E} ^{2}<\| u_{E X}-u_{F E}+\left.v\right|_{E} ^{2} \\
& \Rightarrow|| u_{E X}-\left.u_{F E}\right|_{E} ^{2}<\| u_{E X}-\left.u\right|_{E} ^{2} \\
& \Rightarrow|| u_{E X}-\left.u_{F E}\right|_{E}=\min || u_{E X}-\left.u\right|_{E}
\end{aligned}
$$

Hence proved.

This theorem shows that the selection $S(I)$ is of crucial importance, since the error of approximation is determined by $S(I)$. This theorem also shows that if $u_{E X}$ happens to lie in $S(I)$ then $u_{F E}=u_{E X}$. Furthermore, the theorem shows that if we construct a sequence of finite element spaces $S_{1} \subset S_{2} \subset \ldots \subset S_{n}$ and compute the corresponding finite element solutions $u(1)_{\mathrm{FE}}, u(2)_{F E}, \ldots, u(n)_{F E}$ then the error measured in the energy norm will decrease monotonically with respect to increasing $n$.

\section{Numerical Examples and Results}

In this section, we consider four nonlinear problems to verify the proposed method described in section 3. All the computations are performed by MATLAB. The error of the approximate solutions are estimated by

$$
|u(x)-\tilde{u}(x)| \text { and }\left|\frac{u(x)-\widetilde{u}(x)}{u(x)}\right|
$$

Where $u(x)$ is the exact solution and $\tilde{u}(x)$ is the approximate solution.

\section{Example 1:}

Here we consider a nonlinear boundary value problem with Diritchlet Boundary Conditions. This problem arises in the finite deflections of an elastic string under a transverse load [9]

$$
\frac{d^{2} u}{d x^{2}}=-\left(1+\sigma^{2}\left(\frac{d u}{d x}\right)^{2}\right), \quad 0 \leq x \leq 1
$$




$$
u(0)=0 \text { and } u(1)=0
$$

The exact solution is

$$
u(x)=\frac{1}{\sigma^{2}} \ln \left(\frac{\cos \sigma\left(x-\frac{1}{2}\right)}{\cos \sigma / 2}\right)
$$

Now using the proposed method illustrated in section 3, we find the system of nonlinear equation

$$
[K+L]\{a\}=\{F\}
$$

where the entries of $K, L$ and $F$ are given by

$$
\begin{aligned}
& K_{i, j}^{[e]}=\int_{[e]}\left[\frac{d \phi_{i}}{d x} \frac{d \phi_{j}}{d x}\right] d x \\
& L_{i, j}^{[e]}=-\int_{[e]}\left[\sigma^{2} \phi_{i} \frac{d \phi_{j}}{d x} \sum_{k=1}^{n} a_{k} \phi_{k}\right] d x \\
& F_{i}^{[e]}=\int_{[e]} \phi_{i}(x) d x+\left[\phi_{i} \frac{d \tilde{u}}{d x}\right]_{[e]}
\end{aligned}
$$

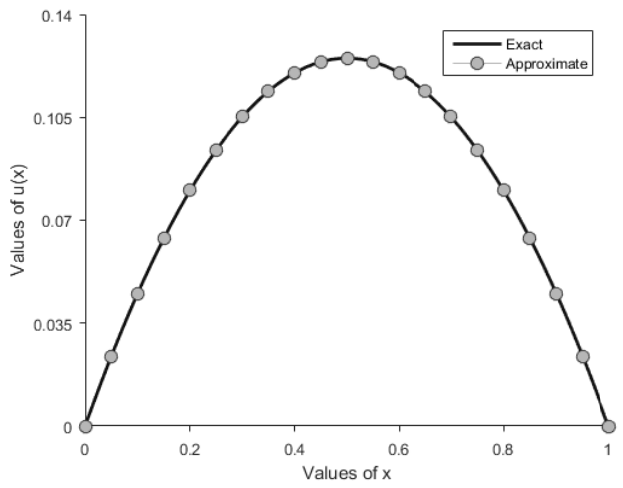

Fig 2: Graphical representation of exact and approximate solution.

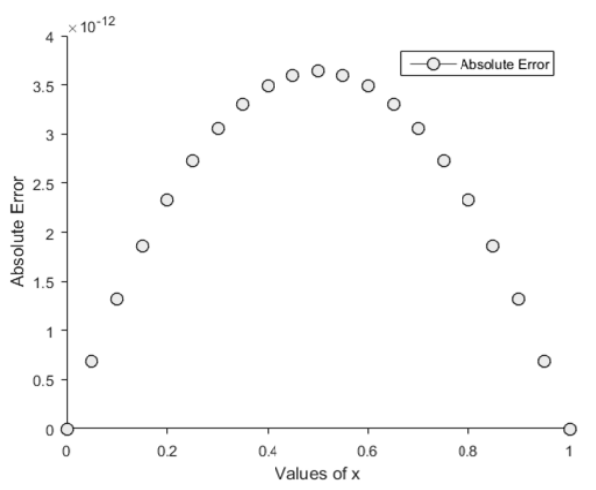

Fig 3: A plot of absolute error of the approximate solutions.

Here we use $\sigma=\frac{1}{7}$. Using 20 quadratic elements and 10 iterations for example 1, we find the results presented in Fig 2 and 3. Cuomo and Marasco [9] found maximum accuracy $2 \times 10^{-7}$ by using finite difference method.

\section{Example 2:}

Now we consider a nonlinear boundary value problem with Neumann Boundary Conditions. This equation is the well-known Burgers' Equation. The one dimensional Burgers' equation is as follows [12] 


$$
\begin{aligned}
& u^{\prime \prime}+u u^{\prime}+u \frac{1}{2} \sin (2 x) \quad 0 \leq x \leq \frac{\pi}{2} \\
& u^{\prime}(0)=1 \text { and } u^{\prime}\left(\frac{\pi}{2}\right)=0
\end{aligned}
$$

The exact solution of this problem is

$u(x)=\sin (x)$

By applying GFEM with 30 quadratic elements and 15 iterations, we find the following results that is shown in Fig 4 and 5.

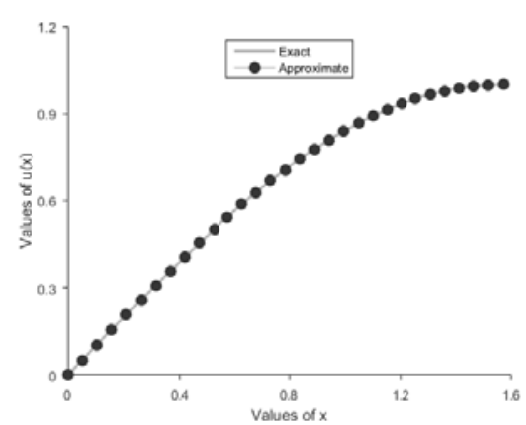

Fig 4: Graphical representation of exact and approximate solutions of example 2 .

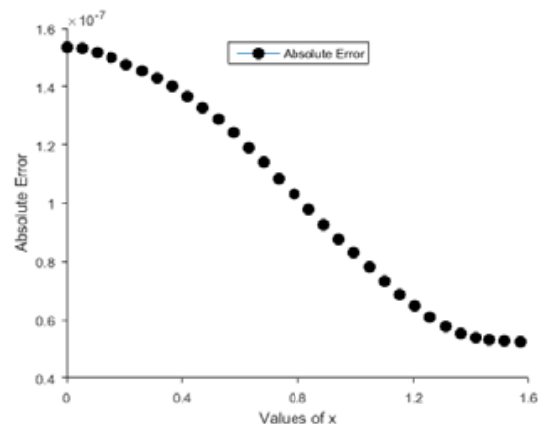

Fig 5: A plot of absolute error of the exact and approximate solution.

\section{Example 3:}

Here we consider a nonlinear boundary value problem with the Robin Boundary Conditions [7].

$$
\begin{aligned}
& \frac{d^{2} u}{d x^{2}}=\frac{1}{2}(1+x+u)^{3}, \quad 0<x<1 \\
& u^{\prime}(0)-u(0)=-\frac{1}{2} \text { and } u^{\prime}(1)+u(1)=1
\end{aligned}
$$

The exact solution of the problem is given by $u(x)=\frac{2}{2-x}-x-1$

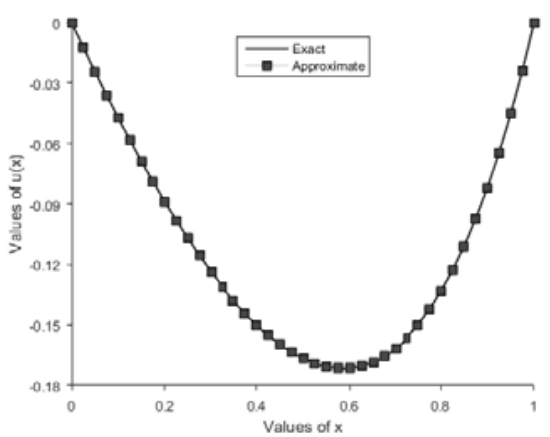

Fig 6: Graphical representation of exact and approximate solutions.

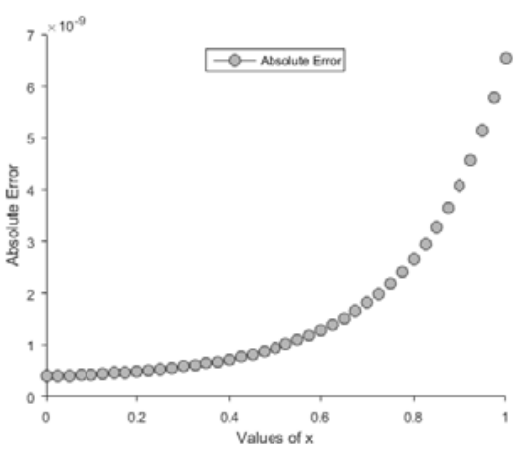

Fig 7: A plot of absolute error of the exact and approximate solution. 
By applying GFEM with 30 quadratic elements and 10 iterations, we find the following results that is shown in Fig 6 and 7. The maximum accuracy obtained by Islam and Shirin [7] by using Galerkin Method with the help 10 Bernoulli polynomials and 8 iterations is $1.508438 \times 10^{-09}$.

\section{Example 4:}

Now we consider an eigenvalue problem [10]

$u^{\prime \prime}+\lambda e^{u}=0, \quad 0<x<1$

Whose boundary conditions are

$u(0)=u(1)=1$

This is the famous one dimensional Bratu's boundary value problem which is of great interest in Magneto hydrodynamics.

If we put $\lambda=-1$ in equation (28a), then the exact solution of this problem is

$u(x)=-\ln 2+\ln (\gamma(x))$

Where

$\gamma(x)=\left\{\operatorname{cosec}\left(\frac{c(2 x-1)}{4}\right)\right\}^{2}$

And $\mathrm{c}$ is the root of the equation $\left[\operatorname{cosec}\left(\frac{c}{4}\right)\right]^{2}=2$

Normally c lies between 0 and $\frac{\pi}{2}$. Here we use $c=1.336055695$.

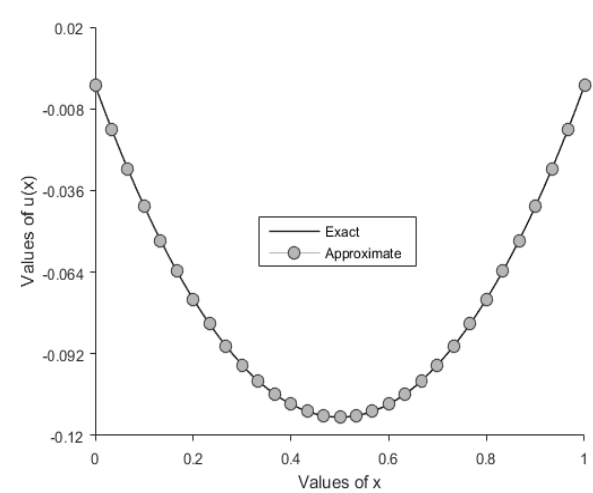

Fig 8: Graphical representation of exact an approximate solutions.

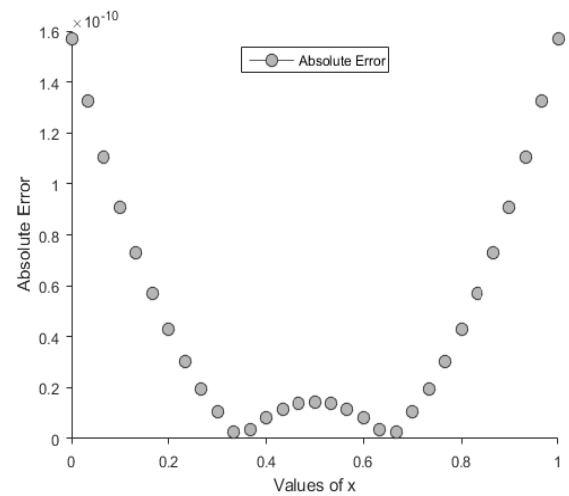

Fig 9: A plot of absolute error of the exact and approximate solution. 
The results are depicted in Fig 8 and 9. We can see that Wazwaz [10] found maximum accuracy $2.48497479 \times 10^{-7}$ by taking up to 14 terms of his series solution where we find maximum accuracy $1.32498612 \times 10^{-12}$.

\section{Conclusion}

In this paper, we have provided a detail formulation for Generalized Galerkin finite element method for the case of both linear and nonlinear boundary value problems with convergence analysis. We have also given the solutions of three nonlinear second order BVPs with Diritchlet, Neumann and Robin boundary conditions and one nonlinear Eigen Value problem. The method has been applied directly without using the linearization or any other restrictive assumptions. In each example, we have compared the approximate results obtained by the proposed method with the exact solution and have found an excellent agreement. All results are depicted graphically as well as in tabular form. From these results, we can conclude that GFEM can be applied as a general technique to find the numerical solution of nonlinear BVPs instead of finite difference method, Galerkin method and Collocation method with haar wavelets. This proposed method can also be applied to solve the higher order nonlinear BVPs and partial differential equations.

\section{Acknowledgement}

The authors are grateful to the learned referee for his valuable comments and suggestions to enrich the quality and improvement of the first version of this manuscript. The first author is also grateful to the Ministry of Science and Technology for granting National Science and Technology (NST) fellowship during the period of research work.

\section{REFERENCES}

[1] Rao, S. S. The finite element method in engineering. Elsevier, 2010.

[2] Wazwaz, A. M. Adomian decomposition method for a reliable treatment of the Bratu-type equations. Applied Mathematics and Computation, 166 (2005), 652-663.

[3] Burden, R. L., \& Faires, J. D. Numerical analysis. Brooks/Cole, USA, (2010).

[4] Islam, M. S., Ahmed, M., \& Hossain, M. A. Numerical Solutions of IVP using Finite Element Method with Taylor Series. GANIT: Journal of Bangladesh Mathematical Society, 30 (2010), 51-58.

[5] Lewis, P. E., \& Ward, J. P. The finite element method: principles and applications. Wokingham: Addison-Wesley, (1991).

[6] Bhatti, M. I., \& Bracken, P. Solutions of differential equations in a Bernstein polynomial basis. Journal of Computational and Applied Mathematics, 205 (2007), 272-280.

[7] Islam, M., \& Shirin, A. Numerical solutions of a class of second order boundary value problems on using Bernoulli polynomials. Applied Mathematics 2. (2011), 1059- 67.14 
[8] Reddy, J. N. An Introduction to Nonlinear Finite Element Analysis. OUP, Oxford (2014).

[9] Cuomo, S., \& Marasco, A. A numerical approach to nonlinear two-point boundary value problems for ODEs. Computers \& Mathematics with Applications, 55 (2008), 2476-2489.

[10] Wazwaz, A. M The successive differentiation method for solving Bratu equation and Bratu type equations. Romanian Journal of Physics, 61 (2016), 774-783.

[11] Szabo, B. A., \& Babu`ska, I. Finite element analysis. John Wiley Sons (1991).

[12] Aly, E. H., Ebaid, A., \& Rach, R. Advances in the Adomian decomposition method for solving two-point nonlinear boundary value problems with Neumann boundary conditions. Computers \& Mathematics with Applications, 63 (2012), 1056-1065.

[13] Aziz, I., \& Sarler, B. The numerical solution of second-order boundary value problems by collocation method with the Haar wavelets. Mathematical and Computer Modelling, 52 (2010), 1577-1590.

[14] Na, T. Y. Computational Methods in Engineering Boundary Value Problems (1979). Academic, New York. 